\title{
A perspective on SARS-CoV-2 and community transmission in the top COVID-19 affected nations
}

\author{
Rini Chaturvedi ${ }^{1}$, Jyoti Chhibber-Goel ${ }^{1}$, , Sumit Malhotra ${ }^{2}$, Amit Sharma ${ }^{1}$ \\ ${ }^{1}$ International Centre for Genetic Engineering and Biotechnology, New Delhi, India, ${ }^{2}$ Centre for Community Medicine, All India Institute of Medical \\ Sciences, New Delhi, India \\ Keywords: sars-cov-2, covid-19, community transmission
}

https://doi.org/10.29392/001c.27141

\section{Journal of Global Health Reports}

Vol. 5, 2021

\begin{abstract}
In most countries, during the initial months of the COVID-19 outbreak resources were directed to mitigation measures that prevented severe acute respiratory syndrome coronavirus 2 (SARS-CoV-2) transmission from symptomatic individuals. The coverage of the pre-symptomatic and asymptomatic individuals' testing required intensive clinical sampling along with rigorous symptom-based screening. Based on the SARS-CoV-2 transmission, the disease outbreak across nations was divided into four distinct stages: (i) epidemic, (ii) community transmission, (iii) local transmission, and (iv) imported cases. Here we discuss the COVID-19 community transmission stage for the top ten COVID-19 affected nations. Epidemic dynamics and policies implemented to contain the spread of SARS-CoV-2 infection varied globally. Further, the mitigation strategies and related health policies for dealing with the pandemic were based on the stages of transmission of the disease. We suggest that correctly identifying the transmission stage during a pandemic ensures the implementation of mitigation strategies. Thus prompt analysis of the status of COVID-19 transmission stage(s) in any nation seems crucial to direct health policies accordingly.
\end{abstract}

The COVID-19 disease is predominantly a respiratory infection that is transmitted via virus-laden respiratory droplets, direct contact, and airborne particles. However, unlike the closely related viral infections, relying only on symptom-based screening has not fully contained severe acute respiratory syndrome coronavirus 2 (SARS-CoV-2) transmission. This was attributed to the viral transmission from asymptomatic, pre-symptomatic (individuals before the onset of symptoms) and the clinically mild infectious population. ${ }^{1}$ Based on the SARS-CoV-2 transmission, the COVID-19 screening and subsequent disease transmission across nations have been divided into four distinct stages: (i) imported cases (transmitted by people traveling from abroad), (ii) local transmission (transmitted to people in contact with others with travel history), (iii) community transmission (transmitted to people who have not been in contact with anyone with a travel history to affected countries and where cases cannot be linked to specific clusters), and (iv) epidemic (when large masses get infected and there is an exponentially rising number of confirmed cases/ deaths).$^{2}$ Establishing the stage of disease transmission is critical to outlining mitigation strategies in order to stem the disease and protect the most vulnerable segments of the population. Accordingly, the disease alleviation policies re- quire analysis of data spanning a wide range of population and covering the various health system efforts in place from each community/society. These data provide an overview and prevent scenarios where the public healthcare system capacity is overburdened during the pandemic in any nation. Here we focus on the third stage of transmission called community transmission that has been described by the World Health Organization (WHO) as "evidenced by the inability to relate confirmed cases through chains of transmission for a large number of cases, or by increasing positive tests through sentinel samples (routine systematic testing of respiratory samples from established laboratories." 2 The Centre for disease control and prevention (CDC, USA) has defined community transmission as, "spread of an illness for which the source of infection is unknown." 3 We focus on the possibility of community transmission and its timelines for few of the top ten COVID-19 afflicted nations.

\section{COMMUNITY TRANSMISSION TIMELINES}

COVID-19 outbreak first appeared in China (Wuhan) in December 2019, and community transmission developed within three months. ${ }^{4}$ By strategically implementing healthcare and containment policies within a short span of

\footnotetext{
a Equal contribution author.

b Equal contribution author.
} 
eleven months, China was able to stem community transmission. 5,6 Once the COVID-19 spread worldwide, cases were reported across nations. The first instance of SARSCoV-2 infection in the United States was reported on January 15,2020 , from Washington. ${ }^{7}$ The CDC team collected multiple samples from four distinct regions and on February 26, 2020 reported the first confirmed case of SARSCoV-2 community transmission from California. ${ }^{8}$ The concerned authorities in California reported SARS-CoV-2 infection in a patient with no travel history (international) or via contact with someone with a travel history or COVID-19 infection. ${ }^{8}$ The SARS-CoV-2 genetic sequences were analyzed via: (i) retroactive testing of stored samples of influenza, (ii) review of the COVID-19 cases reported early from the emergency rooms, (iii) discovery of two people in California that succumbed to COVID-19 in January 2020, and (iv) an infected patient from San Francisco who died on February 2020. Based on these data, CDC concluded that community transmission began in the USA in late January or early February $2020 .{ }^{9}$ Brazil reported its first SARSCoV-2 positive case on 25 February 2020 in São Paulo, and subsequently on 13 March 2020 community transmission was reported in São Paulo and Rio de Janeiro states. ${ }^{10}$ France reported its first case on 24 January 2020, and community transmission was reported by 4 April 2020. Turkey reported its first case on 10 March 2020 and within 30 days, Turkey appeared in the list of countries with community transmission. ${ }^{11,12}$ By the end of April 2020, other countries like Germany, Spain, and eastern European countries had confirmed community transmission as well. ${ }^{13,14}$

\section{GUIDELINES TOWARDS THE MANAGEMENT OF COMMUNITY TRANSMISSION}

The mitigation strategies and related health policies for dealing with the pandemic vary based on the stages of disease transmission. During the first two stages of transmission, i.e. in imported cases and local transmission, contact tracing, individual case identification, and quarantine are needed. The actions to manage the third stage called community transmission differ greatly. During community transmission, the agent is transmitted to people who may not have been in contact with anyone with a travel history to affected countries and where cases cannot be linked to specific clusters. Surveillance at this stage focuses on tracking trends in afflicted populations, the impact on healthcare services, geographical dispersion, transmission intensity, and virological characteristics. This multisource provides ongoing risk evaluations so that necessary public health measures can be taken. During the fourth stage of an epidemic, significant numbers of people become affected, and the number of confirmed cases/deaths increases exponentially. Government resources should be focused on monitoring the spread, identifying and managing severe cases, preventing virus' onward transmission, alleviating strains on healthcare services, advising the public and reducing overall socio-economic impact when dealing with large-scale COVID-19 community transmission. Border closures, for example, may be of little utility once large-scale community transmission has been established, therefore, their effectiveness should be weighed against the social and financial costs.

To manage and suppress the community transmission of COVID-19, countries incorporated specific guidelines to combat and prevent it. These guidelines include: (i) identification and prevention as well as analyses of the workplace health and safety risks to ensure smooth functioning of essential services and healthcare systems, (ii) collaborations between organizations from multiple sectors like health, social, environmental in response to public health emergency as deemed fit, (iii) surveillance and risk assessment, and (iv) efficient testing, clinical management, and sufficient placement of the healthcare services for the vulnerable and infected individuals. ${ }^{15}$ These steps partly ensured that the nations tackling this pandemic could focus their resources on monitoring the geographical spread and transmission of the virus. The identification and management of severe cases were also central, and limited lockdowns prevented onward transmission with an aim to alleviate the strain placed on healthcare services. These steps possibly helped to reduce the overall social and economic impact on the citizens and nation as a whole. Therefore, as community transmission spreads individual quarantine/ isolation, contact tracing is no longer efficient, necessary, and/or effective. Instead, surveillance is focused on monitoring trends for geographical spread, transmission intensity, affected populations, virological features, and impacts on healthcare services. Large-scale vaccination drives as adopted for polio elimination are one of the mitigation steps to control the community transmission for the rapid spread of the infection. ${ }^{16}$

\section{CONCLUSIONS}

This work emphasizes the importance of understanding mitigation steps to be taken once community transmission has been reached so as to avoid the fourth stage of an epidemic. Pandemic mitigation policies to contain the spread of SARS-CoV-2 infection vary globally. Thus, the crosscountry comparisons of the policies implemented worldwide may only be partially useful owing to the varied socioeconomic impact of the mitigation strategies as well as the differences in healthcare systems. Countries that have suffered during the previous pandemics like SARS and MERS have more successfully contained SARS-CoV-2 by taking extensive preventive measures. For instance, Singapore contained the transmission of SARS-CoV-2 within a span of four months. ${ }^{17}$ Despite declining cases, Singapore continued with extensive testing and on 18th May 2021 reported 27 new cases that were attributed to community transmission. Concerned authorities in Singapore initiated control of the rise in COVID-19 cases as data on new emerging viral variants emerged. ${ }^{18}$ Thus it is vital that the pandemic stages are robustly assessed and accepted in nations so that appropriate mitigation measures can be taken and planned.

In the community transmission stage, contact tracing and efforts to increase testing facilities become less useful as this can result in deviation of resources. During community transmission, priority is often given to treatment and strengthening of the healthcare facilities for saving the vulnerable in the society. Many nations with SARS-CoV-2 
infections who had reached the 'community transmission' stage took heed and implemented measures for preventing uncontrolled fatalities during the successive waves of COVID-19, largely by vaccination and by strengthening their healthcare facilities. Failure to alert the communities can result in varied risk perceptions in the communities. Hence, it can be considered important to label community transmission in the regions where it is occurring.

\section{FUNDING}

None.

\section{AUTHORSHIP CONTRIBUTIONS}

All authors contributed to the manuscript.

\section{COMPETING INTERESTS}

The authors completed the Unified Competing Interest form at www.icmje.org/disclosure-of-interest/ (available upon request from the corresponding author) and declare no conflicts of interest.

\section{CORRESPONDENCE TO:}

Jyoti Chhibber-Goel, Molecular Medicine, International Centre for Genetic Engineering and Biotechnology, New Delhi, India. jyotichhibbergoel@gmail.com

Submitted: June 18, 2021 GMT, Accepted: July 28, 2021 GMT 


\section{REFERENCES}

1. Malhotra S, Rahi M, Das P, et al. Epidemiological profiles and associated risk factors of SARS-CoV-2 positive patients based on a high-throughput testing facility in India. Open Biol. 2021;11(6):200288. doi:1 $\underline{0.1098 / \text { rsob.200288 }}$

2. World Health Organization. Considerations for implementing and adjusting public health and social measures in the context of COVID-19. Published 2020. https://apps.who.int/iris/bitstream/handle/1066 5/336374/WHO-2019-nCoV-Adjusting_PH_measure s-2020.2-eng.pdf? sequence $=1$ \&isAllowed $=y$

3. Centers for Disease Control and Prevention. Implementation of Mitigation Strategies for Communities with Local COVID-19 Transmission. Published 2021. https://www.cdc.gov/coronavirus/201 9-ncov/community/community-mitigation.html

4. Liu J, Liao X, Qian S, et al. Community Transmission of Severe Acute Respiratory Syndrome Coronavirus 2, Shenzhen, China, 2020. Emerg Infect Dis. 2020;26(6):1320-1323. doi:10.3201/eid2606.2002 $\underline{39}$

5. Zou H, Shu Y, Feng T. How Shenzhen, China avoided widespread community transmission: a potential model for successful prevention and control of COVID-19. Infect Dis Poverty. 2020;9(1):89. doi:10.1 186/s40249-020-00714-2

6. Risk of COVID-19 community transmission "basically eliminated": Chinese mayor. Business Today. Published October 17, 2020. https://www.busi nesstoday.in/current/world/risk-of-covid-19-commun ity-transmission-basically-eliminated-chinese-mayo r/story/419241.html

7. Holshue ML, DeBolt C, Lindquist S, et al. First Case of 2019 Novel Coronavirus in the United States. $N$ Engl J Med. 2020;382(10):929-936. doi:10.1056/NEJMo a2001191

8. Williams S. First US Case of Apparent SARS-CoV-2 Community Transmission. Published online February 27, 2020. https://www.the-scientist.com/news-opinio n/first-us-case-of-apparent-sars-cov-2-community-tr ansmission-67196

9. Branswell H. When did the coronavirus start spreading in the U.S.? Likely in January, CDC analysis suggests. STAT. Published online May 29, 2020. http s://www.statnews.com/2020/05/29/cdc-local-transmis sion-coronavirus-united-states/
10. Coelho FC, Lana RM, Cruz OG, et al. Assessing the spread of COVID-19 in Brazil: Mobility, morbidity and social vulnerability. PLoS ONE. 2020;15(9):e0238214. doi:10.1371/journal.pone.0238214

11. Cakir B. COVID-19 in Turkey: Lessons Learned. J Epidemiol Glob Health. 2020;10(2):115-117. doi:10.299 1/jegh.k.200520.001

12. Coronavirus Disease 2019 (COVID-19) Situation Report - 101.; 2020. https://www.who.int/docs/defaul t-source/coronaviruse/situation-reports/20200430-sit rep-101-covid-19.pdf?sfvrsn=2ba4e093_2

13. Torri E, Sbrogiò LG, Rosa ED, Cinquetti S, Francia F, Ferro A. Italian Public Health Response to the COVID-19 Pandemic: Case Report from the Field, Insights and Challenges for the Department of Prevention. Int J Environ Res Public Health. 2020;17(10):3666. doi:10.3390/ijerph17103666

14. Eastern Europe/Russia: COVID-19 transmission continues throughout eastern Europe and Russia in April /update 9. Accessed May 18, 2021. https://ww w.garda.com/crisis24/news-alerts/469876/eastern-eur operussia-covid-19-transmission-continues-through out-eastern-europe-and-russia-in-april-update- 9

15. Framework for Implementation of COVID-19 Community Mitigation Measures for Lower-Resource Countries . Published June 26, 2020. https://www.cd c.gov/coronavirus/2019-ncov/global-covid-19/commu nity-mitigation-measures.html

16. Rahi M, Sharma A. Mass vaccination against COVID-19 may require replays of the polio vaccination drives. E Clinical Medicine. 2020;25:100501. doi:10.1016/j.eclinm.2020.100501

17. Chaturvedi R, Malhotra S, Sharma A. Epidemiological profiles of SARS-CoV and SARSCov-2 in Singapore and its promising containment strategies. J Glob Health. 2021;11:03027. doi:10.7189/i ogh.11.03027

18. Singh J, Rahman SA, Ehtesham NZ, Hira S, Hasnain SE. SARS-CoV-2 variants of concern are emerging in India. Nat Med. 2021;27(7):1131-1133. $\underline{\mathrm{d}}$ oi:10.1038/s41591-021-01397-4 